\title{
Operator algebra for propagation problems involving phase conjugation and nonreciprocal elements
}

\author{
Amnon Yariv
}

\begin{abstract}
A self-consistent formalism is developed for treating propagation of beams in situations which include phase conjugation and nonreciprocal elements. Two equivalent field representations, the rectangular polarization and the circular polarization representation, are considered, and the rules for transforming between them are derived. An example involving a proposed new current fiber sensor is analyzed using the formalism.
\end{abstract}

\section{Introduction}

We have recently been led to a consideration of propagation problems in situations which involve a combination of optical media, both reciprocal and nonreciprocal, as well as phase conjugate reflection. These problems required the consistent application of certain rules which are derived in what follows.

Since many of the problems involve propagation through magnetic media exhibiting Faraday rotation, it is convenient to use either circularly polarized (CP) or rectangular polarization $(\mathrm{RP})$ representations of the transverse electric field vector and to switch freely from one representation to the other. We adopt a convention whereby a wave propagates always in the $+z$ direction so that the $z$ direction after a phase conjugate reflection is the negative of the $z$-direction before reflection. If the propagation is in a fiber, the $z$ axis is chosen as the local direction of propagation.

A given transverse field vector (field) will be denoted by either a rectangular polarization (RP) representa-

The author is with California Institute of Technology, Division of Engineering \& Applied Science, Pasadena, California 91125.

Received 21 October 1986.

0003-6935/87/214538-03\$02.00/0.

(C) 1987 Optical Society of America. tion vector with components $(a, b)$ or a circularly polarized (CP) representation $(A, B)$. We use curly and square brackets to represent the field vector in the CP and $\mathrm{RP}$ representations, respectively:

$$
\begin{aligned}
\mathbf{E}_{t} & =\left\{\begin{array}{l}
A \\
B
\end{array}\right\} \text { in (CP), } \\
\mathbf{E}_{t} & =\left[\begin{array}{l}
a \\
b
\end{array}\right] \text { in (RP). }
\end{aligned}
$$

Our convention is such that $\left\{{ }_{0}^{1}\right\}$ corresponds to a unit vector seen rotating in the ccw sense by an observer gazing along the $+z$ axis. These two representations are related by the transformations ${ }^{1}$

$$
\begin{aligned}
& \left\{\begin{array}{l}
A \\
B
\end{array}\right\}=\frac{1}{\sqrt{2}}\left|\begin{array}{ll}
1 & -i \\
1 & +i
\end{array}\right|\left[\begin{array}{l}
a \\
b
\end{array}\right] \equiv \mathbf{T}\left[\begin{array}{l}
a \\
b
\end{array}\right], \\
& {\left[\begin{array}{l}
a \\
b
\end{array}\right]=\frac{1}{\sqrt{2}}\left|\begin{array}{ll}
1 & 1 \\
i & -i
\end{array}\right|\left\{\begin{array}{l}
A \\
B
\end{array}\right\} \equiv \mathbf{S}\left\{\begin{array}{l}
A \\
B
\end{array}\right\},}
\end{aligned}
$$

so that $\mathbf{S}=\mathbf{T}^{-1}$.

To transform from a representation of the RP field vector in the incident coordinate system to the reflected system we choose without loss of generality to let $x$ $\rightarrow-x$ and $z \rightarrow-z$ so that

$$
\left[\begin{array}{l}
a^{\prime} \\
b^{\prime}
\end{array}\right]=\left|\begin{array}{ll}
-1 & 0 \\
0 & 1
\end{array}\right| \overrightarrow{\left[\begin{array}{l}
a \\
b
\end{array}\right]} \equiv \mathbf{r}\left[\begin{array}{l}
a \\
b
\end{array}\right]=\left[\begin{array}{l}
\overrightarrow{-a} \\
b
\end{array}\right],
$$

where the top arrow indicates the direction. Likewise in the $\mathrm{CP}$ representation

$$
\left\{\begin{array}{l}
\overrightarrow{A^{\prime}} \\
B^{\prime}
\end{array}\right\}=\left\{\begin{array}{ll}
0 & -1 \\
-1 & 0
\end{array}\right\}\left\{\begin{array}{l}
\vec{A} \\
B
\end{array}\right\} \equiv \mathbf{R}\left\{\begin{array}{l}
\vec{A} \\
B
\end{array}\right\}=\left\{\begin{array}{l}
\overleftarrow{-B} \\
-A
\end{array}\right\} .
$$

From Refs. 3 and $4 \mathbf{r}=\mathbf{r}^{-1}$ and $\mathbf{R}=\mathbf{R}^{-1}$.

We next develop the matrix representations of certain common optical components and media.

\section{Retardation Plates}

The matrix representation of a plate with a retardation $\alpha$ rotated by an angle $\theta$ from the $x$ axis is ${ }^{1,2}$ 


$$
\vec{\gamma}_{\theta, \alpha}^{(\mathrm{RP})}=\left|\begin{array}{lr}
\cos ^{2} \theta \exp (i \alpha / 2)+\sin ^{2} \theta \exp (-i \alpha / 2) & i \sin 2 \theta \sin \frac{\alpha}{2} \\
i \sin 2 \theta \sin \frac{\alpha}{2} & \sin ^{2} \theta \exp (i \alpha / 2)+\cos ^{2} \theta \exp (-i \alpha / 2)
\end{array}\right|
$$

in the incident coordinate system in the $\mathrm{RP}$ representation. For the common special case of $\theta=\pi / 4$

$$
\vec{\gamma}_{\pi / 4, \alpha}^{(\mathrm{RP})}=\left|\begin{array}{lr}
\cos \frac{\alpha}{2} & i \sin \frac{\alpha}{2} \\
i \sin \frac{\alpha}{2} & \cos \frac{\alpha}{2}
\end{array}\right| .
$$

To find the representation of any operator in the reflected frame we use

$$
\overleftarrow{\mathbf{O}}=\mathbf{r} \overrightarrow{\mathbf{O}} \mathbf{r}^{-1} \text {. }
$$

According to Eq. (7) $\overline{\mathbf{O}}$ is obtained from $\overrightarrow{\mathbf{O}}$ by reversing the signs of the off-diagonal elements. Applying Eq. (7) to Eq. (6), for example, leads to

$$
\overleftarrow{\gamma}_{\pi / 4, \alpha}^{\mathrm{RP})}=\left|\begin{array}{lr}
\cos \frac{\alpha}{2} & -i \sin \frac{\alpha}{2} \\
-i \sin \frac{\alpha}{2} & \cos \frac{\alpha}{2}
\end{array}\right|
$$

To find the matrix representation of retardation plates in CP space we use ${ }^{2}$

$$
\begin{aligned}
\vec{\gamma}_{\pi / 4, \alpha}^{(\mathrm{CP})} & =\mathbf{T} \vec{\gamma}_{\pi / 4, \alpha}^{(\mathrm{RP})} T^{-1} \\
& =\left\{\begin{array}{ll}
\cos \frac{\alpha}{2} & \sin \frac{\alpha}{2} \\
-\sin \frac{\alpha}{2} & \cos \frac{\alpha}{2}
\end{array}\right\},
\end{aligned}
$$

and from Eq. (4)

$$
\bar{\gamma}_{\pi / 4, \alpha}^{(\mathrm{CP})}=\mathbf{R} \vec{\gamma}_{\pi / 4, \alpha}^{(\mathrm{CP})} \mathbf{R}^{-1}=\left\{\begin{array}{rr}
\cos \frac{\alpha}{2} & -\sin \frac{\alpha}{2} \\
\sin \frac{\alpha}{2} & \cos \frac{\alpha}{2}
\end{array}\right\} .
$$

As a check on our internal consistency we find that we could have also derived the last result from

$$
\overleftarrow{\gamma}_{\pi / 4, \alpha}^{(\mathrm{CP})}=\mathbf{T} \tilde{\gamma}_{\pi / 4, \alpha}^{(\mathrm{RP})} \mathbf{T}^{-1}
$$

\section{Faraday Rotation}

Here we start with the CP representation which is more natural for situations involving Faraday rotation since the eigenvectors of the Faraday medium are pure circularly polarized vectors.

The Faraday matrix for the $\mathrm{CP}$ representation is

$$
\overrightarrow{\mathbf{F}}_{(\theta)}^{(C P)}=\left\{\begin{array}{lr}
\exp (i \theta) & 0 \\
0 & \exp (-i \theta)
\end{array}\right\},
$$

while in the reflected frame the matrix representation of the same element is

$$
\overleftarrow{F}_{(\theta)}^{(\mathrm{CP})}=\mathbf{R} \overrightarrow{\mathbf{F}}^{(\mathrm{CP})} \mathbf{R}^{-1}=\left\{\begin{array}{lr}
\exp (-i \theta) & 0 \\
0 & \exp (i \theta)
\end{array}\right\},
$$

so that $\overrightarrow{\mathrm{F}}(\theta)=\overline{\mathrm{F}}(-\theta)$. We also used $\theta=V H L$ as the Faraday rotation angle ( $V$ is the Verdet constant, $H$ the magnetic field, $L$ the medium length). In the rectangular representation we obtain

$$
\overrightarrow{\mathbf{F}}^{(\mathrm{RP})}=\mathbf{T}^{-1} \overrightarrow{\mathbf{F}}^{(\mathrm{CP})} \mathbf{T}=\left|\begin{array}{ll}
\cos \theta & \sin \theta \\
-\sin \theta & \cos \theta
\end{array}\right|,
$$

i.e., a rotation by $\theta$. In the reflected (RP) frame we obtain

$$
\overleftarrow{\mathbf{F}}^{(\mathrm{RP})}=\mathbf{R} \overrightarrow{\mathbf{F}}^{(\mathrm{RP})} \mathbf{R}^{-1}=\left|\begin{array}{rr}
\cos \theta & -\sin \theta \\
\sin \theta & \cos \theta
\end{array}\right|
$$

\section{Polarizers}

By a simple application of the above techniques we obtain for $x$ polarizers

$$
\mathbf{P}_{x}^{(\mathrm{CP})}=1 / 2\left\{\begin{array}{ll}
1 & 1 \\
1 & 1
\end{array}\right\}
$$

for both incident and reflected frames. In the RP frame

$$
\mathbf{P}_{x}^{(\mathrm{RP})}=\left[\begin{array}{ll}
1 & 0 \\
0 & 0
\end{array}\right],
$$

while for $y$ polarizers

$$
\begin{aligned}
& \mathbf{P}_{y}^{(\mathrm{CP})}=1 / 2\left\{\begin{array}{ll}
1 & -1 \\
-1 & 1
\end{array}\right\}, \\
& \mathbf{P}_{y}^{(\mathrm{RP})}=1 / 2\left[\begin{array}{ll}
0 & 0 \\
0 & 1
\end{array}\right],
\end{aligned}
$$

\section{Phase Conjugate Mirror (PCM)}

The effect of a true (scalar) PCM which renders the complex conjugate of each Cartesian coordinate of a vector would by definition be the transformation

$$
\left[\begin{array}{l}
a \\
b
\end{array}\right] \rightarrow\left[\begin{array}{l}
a^{*} \\
b^{*}
\end{array}\right]
$$

when we use the RP system and the same $z$ axis for the incident wave and the reflected conjugate wave. Since the conjugate wave always travels in the direction opposite to that of the incident wave and since we agreed to adopt the convention of always representing a wave in a coordinate system in which the direction of propagation is designated the $+z$ axis, we will represent the conjugate wave in its new reflected frame by changing the sign of the $x$ (or $y$ ) component. The effect of phase conjugation in the RP frame will then be represented symbolically by

$$
\phi^{*}\left[\begin{array}{l}
a \\
b
\end{array}\right]=\left[\begin{array}{l}
-a^{*} \\
b^{*}
\end{array}\right] .
$$

To determine the effect of phase conjugation on a field in the CP representation, we use Eq. (20) as well as Eqs. (1) and (2):

$$
\phi^{*}\left\{\begin{array}{l}
A \\
B
\end{array}\right\}=\mathbf{T} \phi^{*}\left[S\left\{\begin{array}{l}
A \\
B
\end{array}\right\}\right]=\left\{\begin{array}{l}
-A^{*} \\
-B^{*}
\end{array}\right\} .
$$




\section{Example}

We now possess all the tools needed to analyze problems involving propagation and phase conjugation with arbitrary combinations of elements. We consider as an example the case of the system illustrated in Fig. 1 , which shows a wave propagating in sequence through an $x$ polarizer $P$, a Faraday rotator biased by a magnetic field $H$ possessing a Faraday angle $\theta$, and retardation plate (rotated at $45^{\circ}$ and introducing a retardation of $\alpha$ ). The wave is then reflected by a true phase conjugate mirror and retraces its path.

We launch a wave $\left[\begin{array}{l}a \\ b\end{array}\right]$ at plane 1 and solve for the reflected wave at the same plane:

$$
\left[\begin{array}{l}
a \\
b
\end{array}\right]_{\text {refl. }}=\mathbf{T}^{-1} \mathbf{P}_{x} \overline{\mathbf{F}}(\theta) \bar{\gamma}(\alpha) \overline{\mathbf{F}}(\theta) \phi^{*}\left[\overrightarrow{\mathbf{F}}(\theta) \vec{\gamma}(\alpha) \overrightarrow{\mathbf{F}}(\theta) \mathbf{P}_{x} \mathbf{T}\left[\begin{array}{l}
\vec{a} \\
b
\end{array}\right]_{\text {incid. }}\right] .
$$

The presence of the operators $T^{-1}$ and $T$ in Eq. (22) indicates that we have chosen to carry out the actual calculation in the CP representation so that all the operators appearing in Eq. (22) are in that representation. If we choose to do the calculation in the RP references, we leave out the $T^{-1}$ and $T$ matrices and take all the matrices in the $\mathrm{RP}$ references.

$\left[\begin{array}{l}\left(E_{x}\right)_{\text {refl. }} \\ \left(E_{y}\right)_{\text {refl. }}\end{array}\right]=\left[\begin{array}{ll}+\cos ^{2} \frac{\alpha}{2} \cos 4 \theta-i \sin \alpha \sin 2 \theta+\sin ^{2} \frac{\alpha}{2} & 0 \\ 0 & 0\end{array}\right]\left[\begin{array}{l}\left(E_{x}\right)_{\text {incid. }} \\ \left(E_{y}\right)_{\text {incid. }}\end{array}\right]$,

or considering only the $x$ polarized input and output,

$$
\begin{aligned}
\left(E_{x}\right)_{\mathrm{out}}= & {\left[-\cos ^{2} \frac{\alpha}{2} \cos 4 \theta-\sin ^{2} \frac{\alpha}{2}\right.} \\
& +i \sin \alpha \sin 2 \theta]\left(E_{x}\right)_{\mathrm{in}},
\end{aligned}
$$

where a sign reversal in Eq. (24) was used to return $\left(E_{x}\right)_{\text {out }}$ to the same frame (incident) as $\left(E_{x}\right)_{\text {in. }}$.

The example can serve as a model for a proposed fiber current sensor. In this case the current to be measured is enclosed by the fiber loop and the two Faraday rotators in the figure represent Faraday rotation in the fiber loop. In a perfect fiber without birefringence or modal coupling $\alpha=0$ and Eq. (24) becomes

$$
\left(E_{x}\right)_{\text {out }}=\left(E_{x}\right)_{\text {in }} \cos 4 \theta,
$$

where $2 \theta$ is the total Faraday rotation between the input and the phase conjugate mirror and is thus proportional to the current to be measured. The presence of the birefringent terms (involving $\alpha$ ) in Eq. (24) shows how the coupling between $x$ and $y$ modes, which is present to some degree in any fiber, interferes with the Faraday rotation. 3,4 This coupling is represented in our own model by a birefringent plate with a retardation $\alpha$. Such a plate couples $x$ and $y$ polarized modes of a fiber in the manner described by Eqs. (8) or (9). From Eq. (24) it follows that changes in the strength of such coupling, i.e., changes in $\alpha$, cannot be distinguished from changes in the current, i.e., $\theta$. One solution to this problem, which involves modal dispersal, will be described separately. ${ }^{5,6}$

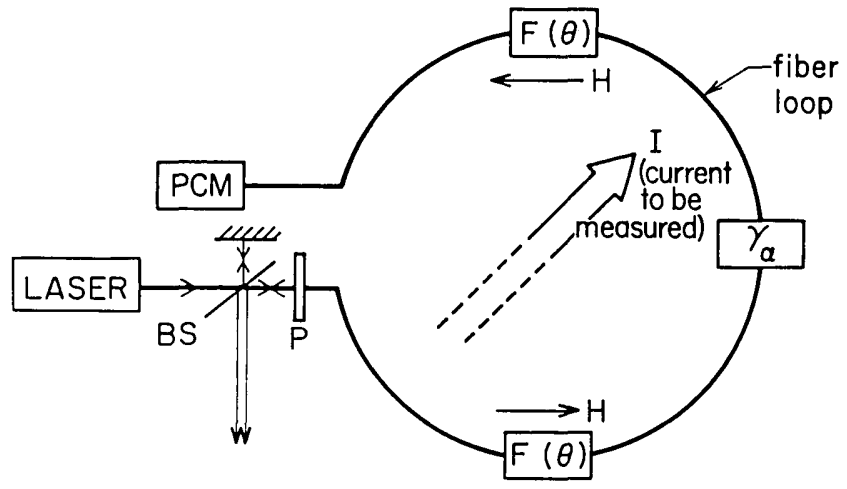

Fig. 1. Schematic diagram of a wave propagating in sequence through an $x$ polarizer $P$, a Faraday rotator $F(\theta)$, retardation plate $\gamma(\alpha)$. It is then reflected by a phase conjugate mirror (PCM) and retraces its path.
The author acknowledges the Army Research Office (R. Guenther) and the Air Force Office of Scientific Research (H. Schlossberg) for the support of this research. Clarifying conversations with S. K. Kwong and Ram Yahalom are gratefully acknowledged.

\section{References}

1. R. C. Jones, "New Calculus for the Treatment of Optical Systems," J. Opt. Soc. Am. 31, 448 (1941). See also A. Yariv and P. Yeh, Optical Waves in Crystals (Wiley, New York, 1984), p. 121.

2. A. Yariv, Quantum Electronics (Wiley, New York, 1968), p. 197.

3. A. Yariv, K. Petermaun, and E. Weidel, "Sensitivity of a FiberOptic Gyroscope to Environmental Magnetic Fields," Opt. Lett. 7, 181, (1982).

4. G. W. Day, D. N. Payne, A. J. Barlow, and J. J. Ramskov-Hansen, "Faraday Rotation in Coiled Monomode Optical Fibers: Isolators, Filters, and Magnetic Sensors," Opt. Lett. 7, 238 (1982).

5. K. Kyuma, A. Yariv, and S. K. Kwong, "Polarization Recovery in Phase Conjugation by Modal Dispersal," Appl. Phys. Lett. 49, 617 (1986).

6. S.-K. Kwong, R. Yahalom, K. Kyuma, and A. Yariv, "Optical Phase Conjugation with Non-Reciprocal Media," submitted for publication (1986). 